\title{
A Modified Method On The Point Spread Function Of Motion Blur Image
}

\author{
Zewei Duan $^{1,}$, Lei Qin ${ }^{2}$ \\ ${ }^{1}$ AVIC Xi'an Aeronautics Computing Technique Research Institute, Xi'an 710056, China; \\ ${ }^{2}$ Xi'an Communications Institute, Xi'an 710106, China. \\ adzw890617@163.com
}

Keywords: Image Restoration, PSF, Direction of Differential Operator, Differential Autocorrelation Algorithm.

\begin{abstract}
In order to effectively restore the motion blurred images, this paper analyzes the point spread function (PSF) under the uniform linear motion module and simplifies the PSF into binary function constituted of blur extent and blur direction. The paper adopts the direction of differential operator based on bilinear interpolation and differential autocorrelation algorithm to estimate the PSF. The experiments demonstrate that the method can precisely discriminate PSF's parameters, the restoration algorithms based on the estimated PSF obtained by the proposed method can effectively restore the experimental blur images.
\end{abstract}

\section{Introduction}

Due to relatively motion between the observed object and imaging devices, the scene energy would abnormally accumulate on the image plane during the filming integral time, this would cause the image motion blur [1]. In practical application, researchers need to restore the blurred images to obtain the well-visual images. The motion blur images exist in daily life, those researches on the estimation of motion blur images' parameters and restoration methods become a hot focus. The image restoration methods can be divided into 2 classes: non-blind image restoration and blind image restoration. In the blind image restoration methods, the restoration methods based on the estimated PSF would be an important one. The paper adopts the direction of differential operator and differential autocorrelation algorithm to estimate the blur parameters. Aiming at testifying the applicability and accuracy of the proposed method to the image restoration, this paper adopts three regularly used restoration algorithms: the constrained least square(CLS) filtering algorithm, the blind convolution(BC) filtering algorithm and Lucy-Richardson(LR) filtering algorithm [2, 3].

\section{Image Restoration Model[4]}

In the image restoration processing, we take blur system as space linear and constant displacement system, the image blur processing can be expressed by the formula as follows:

$$
\mathrm{g}(\mathrm{x}, \mathrm{y})=\mathrm{f}(\mathrm{x}, \mathrm{y}) * \mathrm{~h}(\mathrm{x}, \mathrm{y})+\mathrm{n}(\mathrm{x}, \mathrm{y})
$$

$\mathrm{f}(\mathrm{x}, \mathrm{y})$ represents original input image, $\mathrm{n}(\mathrm{x}, \mathrm{y})$ represents additive Gaussian white noise, $\mathrm{h}(\mathrm{x}, \mathrm{y})$ represents retrograde function, $\mathrm{g}(\mathrm{x}, \mathrm{y})$ represents blurred image, * is linear convolution operator.

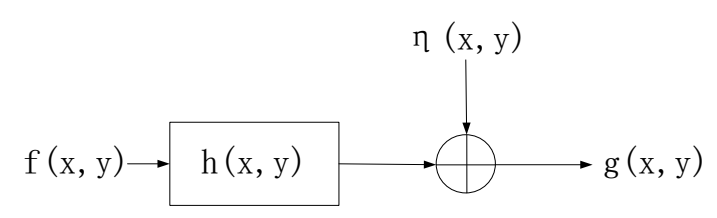

Fig. 1 Classical image retrograde model

In order to simplify the calculation and analysis, the motion in short time can be taken as uniform linear motion. Uniform linear motion is the basis of other motions and also the component of complicated motion, variable motion can be divided into uniform linear motion during short time. 
Thus, among all the motion blur conditions, the restoration problem caused by uniform linear motion possesses universality. Ignoring the noises, uniform linear motion can be taken as the motion object's luminance integration during the exposure time:

$$
\mathrm{g}(\mathrm{x}, \mathrm{y})=\int_{0}^{\mathrm{I}} \mathrm{f}\left[\mathrm{x}-\mathrm{x}_{0}(\mathrm{t}), \mathrm{y}-\mathrm{y}_{0}(\mathrm{t})\right] \mathrm{dt}
$$

The formula is the retrograde model of uniform linear motion. $x_{0}(t), y_{0}(t)$ represents the motion component of original image $\mathrm{f}(\mathrm{x}, \mathrm{y})$ along the $\mathrm{X}$ axis and $\mathrm{Y}$ axis respectively, $\mathrm{T}$ represents acquisition time.

The Fourier transformation of formula (2):

$$
\begin{aligned}
& \mathrm{G}(\mathrm{u}, \mathrm{v})=\int_{-\infty}^{+\infty} \int_{-\infty}^{+\infty} \mathrm{g}(\mathrm{x}, \mathrm{y}) \exp [-\mathrm{j} 2 \pi(\mathrm{ux}+\mathrm{vy})] \mathrm{dxdy} \\
& =\int_{0}^{\mathbb{T}}\left[\int_{-\infty}^{+\infty} \int_{-\infty}^{+\infty} g(x, y) \exp [-j 2 \pi(u x+v y)] d x d y\right] d t \\
& =F(\mathrm{u}, \mathrm{v}) \int_{0}^{\mathbb{I}} \exp \left[-\mathrm{j} 2 \mathrm{~m}\left(\mathrm{ux}_{0}(\mathrm{t})+\mathrm{vy}_{0}(\mathrm{t})\right)\right] \mathrm{dt} \\
& \text { Define } H(u, v)=\int_{0}^{\mathbb{T}} \exp \left[-j 2 \pi\left(u_{0}(t)+v y_{0}(t)\right)\right] d t \\
& \mathrm{G}(\mathrm{u}, \mathrm{v})=\mathrm{F}(\mathrm{u}, \mathrm{v}) \mathrm{H}(\mathrm{u}, \mathrm{v})
\end{aligned}
$$

The formula (4) is the frequency domain's retrograde model of uniform linear motion. According to the formula, if the motion components $x_{0}(t)$ and $y_{0}(t)$ are known, the original image $f(x, y)$ can be restored. Supposed that the motion component along $\mathrm{X}$ axis is the only influence factor and $\mathrm{y}_{0}(\mathrm{t})=0$, the formula (2) can be expressed as:

$\mathrm{g}(\mathrm{x}, \mathrm{y})=\int_{0}^{\mathrm{I}} \mathrm{f}\left[\mathrm{x}-\mathrm{x}_{0}(\mathrm{t}), \mathrm{y}\right] \mathrm{dt}$

Define $\mathrm{L}$ represents the displacement along $\mathrm{X}$ axis, namely blur extent. Because of uniform linear motion, here comes the relationship:

$\mathrm{x}_{0}(\mathrm{t})=\frac{\mathrm{Lt}}{\mathrm{T}}$

$\mathrm{H}(\mathrm{u}, \mathrm{v})$ could be expressed as:

$\mathrm{H}(\mathrm{u}, \mathrm{v})=\int_{0}^{\mathbb{I}} \exp \left[-\mathrm{j} 2 \pi u x_{0}(\mathrm{t})\right] \mathrm{dt}=\int_{0}^{\mathbb{T}} \exp \left[-\mathrm{j} 2 \pi \mathrm{u} \frac{\mathrm{Lt}}{\mathrm{T}}\right] \mathrm{dt}=\frac{\mathrm{T}}{\pi \mathrm{Lu}} \sin (\mathrm{L} \mathrm{Lu}) \exp (-\mathrm{j} \pi \mathrm{Lu})$

According to the inverse Fourier transformation of formula (7), the PSF of uniform linear motion could be obtained:

$$
\mathrm{h}(\mathrm{x}, \mathrm{y})=\left\{\begin{array}{l}
\frac{1}{\mathrm{~L}} \\
0, \text { else }
\end{array}, 0 \leq \mathrm{x} \leq \mathrm{L}\right.
$$

The value range of $\mathrm{x}$ is $0,1,2 \cdots \mathrm{L}-1$. If the influence of motion component along $\mathrm{Y}$ axis is taken into account as well, The PSF can be expressed:

$$
\mathrm{h}(\mathrm{x}, \mathrm{y})\left\{\begin{array}{c}
\frac{1}{\mathrm{~L}} \cdot \sqrt{\mathrm{x}^{2}+\mathrm{y}^{2}} \leq \mathrm{L} \text { and } \frac{x}{y}=-\tan \theta \\
0 \text {, else }
\end{array}\right.
$$

$\theta$ represents the angle between motion direction and $\mathrm{X}$ axis, the value of $\mathrm{y}$ up $\mathrm{X}$ axis is defined plus and the one below $\mathrm{X}$ axis is defined minus.

Thus it can be seen, if both of blur direction and blur extent are known, the PSF can be solved, on the base of a known PSF, the restoration of motion blur image can be achieved.

\section{Estimation of PSF and Image Restoration}

\subsection{Estimation of PSF's Direction[5].}

The statistics of images demonstrate that the original images can be generally taken as isotropy Markov process, the autocorrelation function and the power spectrum of original image are isotropy $[6,7]$. The motion blur reduces the image's high-frequency component along the motion's direction but has less influence on the high-frequency component along other direction. The more divergence angle of the direction, the less impact on the image high-frequency component, the image's high-frequency component which is perpendicular to the motion direction won't be impacted. Taking directional high-pass filtering (directional differential) to the blurred image, the image energy will lose most when the filtering direction is exactly the motion direction because that there is least high-frequency component existing on the direction, the sum of the grey level of the differential 
image will be the minimum. So, the direction corresponding to the minimum sum of image's gray level obtained by direction differential is exactly the motion blur direction.

$\mathrm{g}(\mathrm{i}, \mathrm{j})$ is defined the point of motion blur image, $\mathrm{g}\left(\mathrm{i}^{\mathrm{b}}{ }_{\mathrm{j}} \mathrm{j}^{\mathrm{j}}\right)$ is an image point whose center is $\mathrm{g}(\mathrm{i}, \mathrm{j})$ and radius is $\Delta \mathrm{r}, \Delta \mathrm{r}$ is the direction differential's differential extent. $\mathrm{D}_{\propto}$ is defined as $3 \times 3$ direction differential operator ( the value varies with the angle $\alpha$ ), $\Delta \mathrm{g}\left(\mathrm{i}_{s}, \mathrm{D}_{\Omega}\right.$ is the differential image of motion blur image $g(i, j)$, we have the formula as follows:

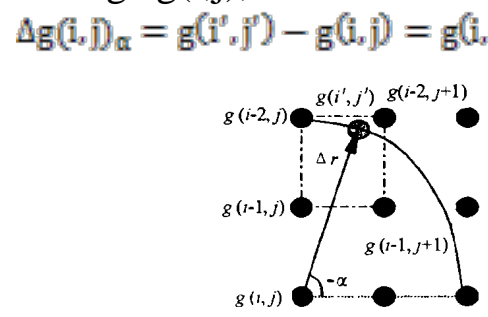

(a) $-\pi / 2 \leqslant \alpha<-\pi / 3$

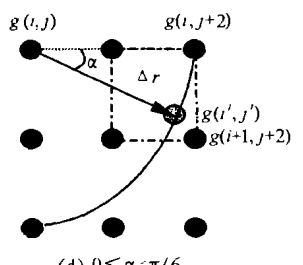

(d) $0 \leqslant x \leqslant \pi / 6$

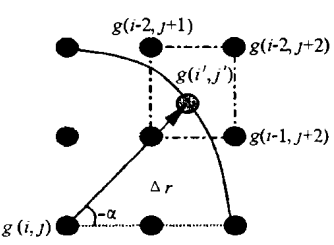

(b) $-\pi / 3 \leqslant \alpha<-\pi / 6$

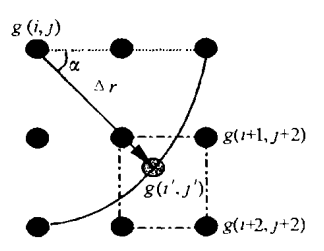

(e) $\pi / 6 \leqslant \alpha \leqslant \pi / 3$

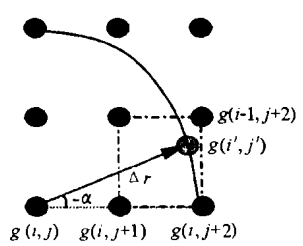

(c) $-\pi ; 6 \leqslant \alpha<0$

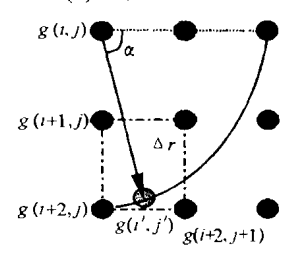

(f) $\pi / 3 \leqslant \alpha-\pi / 2$

Fig. 2 Schematic of $3 \times 3$ direction differential operator

When $-\frac{\pi}{2} \leq \alpha<-\frac{\pi}{a}$

$\mathrm{D}_{\alpha}=\left[\begin{array}{ccc}-1^{2}-2 \sin \alpha+2 \cos \alpha+4 \sin \alpha \cos \alpha & -2 \cos \alpha-4 \sin \alpha \cos \alpha & 0 \\ 2+2 \sin \alpha-4 \cos \alpha-4 \sin \alpha \cos \alpha & 4 \cos \alpha+4 \sin \alpha \cos \alpha & 0 \\ -1 & 0 & 0\end{array}\right]$

When $-\frac{\pi}{a} \leq \alpha<-\frac{\pi}{6}$

$\mathrm{D}_{\alpha}=\left[\begin{array}{ccc}0 & -2-4 \sin \alpha+2 \cos \alpha+4 \sin \alpha \cos \alpha & 1+2 \sin \alpha-2 \cos \alpha-4 \sin \alpha \cos \alpha \\ 0 & 4+4 \sin \alpha-4 \cos \alpha-4 \sin \alpha \cos \alpha & -2-2 \sin \alpha+4 \cos \alpha+4 \sin \alpha \cos \alpha \\ -1 & 0 & 0\end{array}\right]$

When $-\frac{\pi}{6} \leq \alpha<0$

$\mathrm{D}_{\alpha}=\left[\begin{array}{ccc}0 & 0 & 0 \\ 0 & -4 \sin \alpha+4 \sin \alpha \cos \alpha & 2 \sin \alpha-4 \sin \alpha \cos \alpha \\ -1 & 2+4 \sin \alpha-2 \cos \alpha-4 \sin \alpha \cos \alpha & -1-2 \sin \alpha+2 \cos \alpha+4 \sin \alpha \cos \alpha\end{array}\right]$

When $0 \leq \alpha<\frac{\pi}{6}$

$\mathrm{D}_{\alpha}=\left[\begin{array}{ccc}-1 & 2-4 \sin \alpha-2 \cos \alpha+4 \sin \alpha \cos \alpha & -1+2 \sin \alpha+2 \cos \alpha-4 \sin \alpha \cos \alpha \\ 0 & 4 \sin \alpha-4 \sin \alpha \cos \alpha & -2 \sin \alpha+4 \sin \alpha \cos \alpha \\ 0 & 0 & 0\end{array}\right]$

When $\frac{\pi}{6} \leq \alpha<\frac{\pi}{a}$

$\mathrm{D}_{\alpha}=\left[\begin{array}{ccc}-1 & 0 & 0 \\ 0 & 4-4 \sin \alpha-4 \cos \alpha+4 \sin \alpha \cos \alpha & -2+2 \sin \alpha+4 \cos \alpha-4 \sin \alpha \cos \alpha \\ 0 & -2+4 \sin \alpha+2 \cos \alpha-4 \sin \alpha \cos \alpha & 1-2 \sin \alpha-2 \cos \alpha+4 \sin \alpha \cos \alpha\end{array}\right]$

When $\frac{\pi}{a} \leq \alpha<\frac{\pi}{2}$

$\mathrm{D}_{\alpha}=\left[\begin{array}{ccc}-1 & 0 & 0 \\ 2-2 \sin \alpha-4 \cos \alpha+4 \sin \alpha \cos \alpha & 4 \cos \alpha-4 \sin \alpha \cos \alpha & 0 \\ -1+2 \sin \alpha+2 \cos \alpha-4 \sin \alpha \cos \alpha & -2 \cos \alpha+4 \sin \alpha \cos \alpha & 0\end{array}\right]$

The sum of the differential image's gray level is as follows:

$\mathrm{I}(\Delta \mathrm{g})_{\AA}=\sum_{\mathrm{i}=0}^{\mathrm{N}-1} \sum_{\mathrm{j}=0}^{\mathrm{N}-1}\left|\Delta \mathrm{g}(\mathrm{i}, \mathrm{j})_{\Omega}\right|$

The value of $a$ is selected according to a certain step on the range $a \in[-\pi / 2, \pi / 2]$, then $\mathrm{I}(\Delta \mathrm{g})_{\alpha}$ can be obtained and so does the minimum minI $(\Delta \mathrm{g})_{\alpha}$, the angle corresponding to the minimum mini $(\Delta \mathrm{g})_{\Omega}$ is exactly the motion blur direction.

\subsection{Estimation of PSF's extent.}

The problem of motion blur extent can be solved by autocorrelation function based on the differential: 
(1) On the base of estimated motion direction, the blurred image should be rotated and parallel to $\mathrm{X}$ axis so that the 2-D problem can be simplified to 1-D problem (along $\mathrm{X}$ axis).

(2) Calculate the rotated image's differential function along horizontal direction at each row $\Delta \mathrm{g}(\mathrm{i}, \mathrm{j})=\mathrm{gx}(\mathrm{i}, \mathrm{j}-1)-\mathrm{gx}(\mathrm{i}, \mathrm{j})$

$\Delta \mathrm{g}(\mathrm{i}, \mathrm{j})$ represents the differential image, $\mathrm{gx}\left(\mathrm{i}_{1} \mathrm{j}\right)$ represents the rotated motion blur image.

(3) Calculate the differential image's autocorrelation function along horizontal direction at each row

$\Delta \mathrm{gR}(\mathrm{i}, \mathrm{j})=\sum_{\mathrm{k}=1}^{\mathrm{N}} \Delta \mathrm{g}(\mathrm{i}, \mathrm{k}) \cdot \Delta \mathrm{g}(\mathrm{i}, \mathrm{k}+\mathrm{j}), \mathrm{i}=1,2,3 \cdots \mathrm{M} ; \mathrm{j}=1,2,3 \cdots \mathrm{N}$

(4) Sum the autocorrelation function of each row, obtain an identification curve L. $\Delta \mathrm{gS}(\mathrm{j})=\sum_{\mathrm{i}=1}^{\mathrm{M}} \Delta \mathrm{gR}(\mathrm{i}, \mathrm{j})$

(5) Draw the curve L, the distance between the minimum valley and null point is blur extent.

\section{Experiment Result and Estimation}

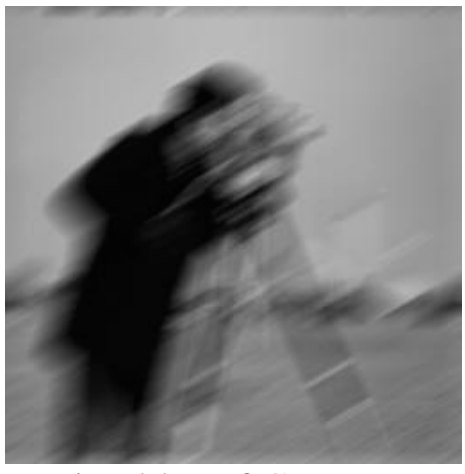

Fig. 3 Motion blur of Cameraman image

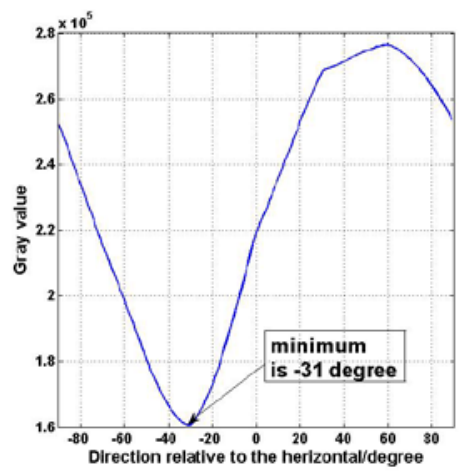

Fig. 4 Blur direction identification

The paper adopts Matlab software(version R2010a) to testify the proposed method. The Fig. 3 is a motion blur image of the classical image named Cameraman, the blur extent is set 30 pixels, the blur direction is set $-30^{\circ}$. The tested motion blur direction obtained by the $3 \times 3$ direction differential operator is $-31^{\circ}$, the error is $1^{\circ}$, the motion blur direction identification curve is as shown in the Fig. 4 . For the original "Cameraman” image's uniform linear motion blur (blur extent 30 pixels) on each direction $\left(-90^{\circ} \sim 90^{\circ}\right)$, the identified result can be obtained by the $3 \times 3$ direction differential operator $\mathrm{D}_{\propto}$ (the step is $\left.1^{5}\right)$, the identified results are shown in the Table 1.the mean value of the identification result is $2^{\circ}$, RMSE is $1.414^{\circ}$, so the identified results are satisfied.

Table 1 Blur direction identification with the $3 \times 3$ direction differential operator ( Unit: degree)

\begin{tabular}{|c|c|c|c|c|c|c|c|c|c|}
\hline Real blur direction & -90 & -80 & -70 & -60 & -50 & -40 & -30 & -20 & -10 \\
\hline Tested blur direction & -89 & -79 & -73 & -64 & -53 & -43 & -31 & -20 & -9 \\
\hline error & 1 & 1 & 3 & 4 & 3 & 3 & 1 & 0 & 1 \\
\hline Real blur direction & 0 & 10 & 20 & 30 & 40 & 50 & 60 & 70 & 80 \\
\hline Tested blur direction & 0 & 7 & 20 & 25 & 37 & 48 & 61 & 72 & 83 \\
\hline error & 0 & 3 & 0 & 5 & 3 & 2 & 1 & 2 & 3 \\
\hline
\end{tabular}

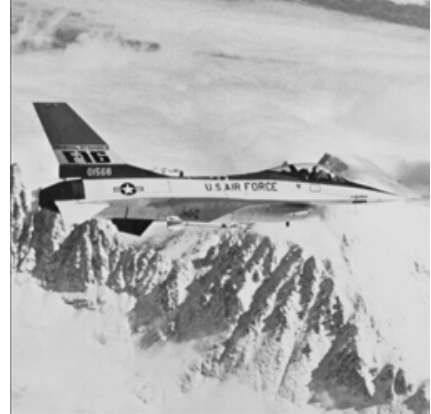

Fig .5 Original image

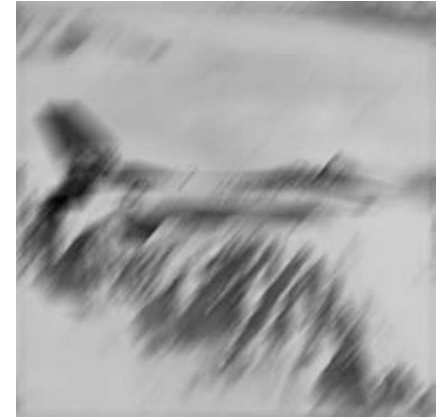

Fig. 6 Motion blur image 


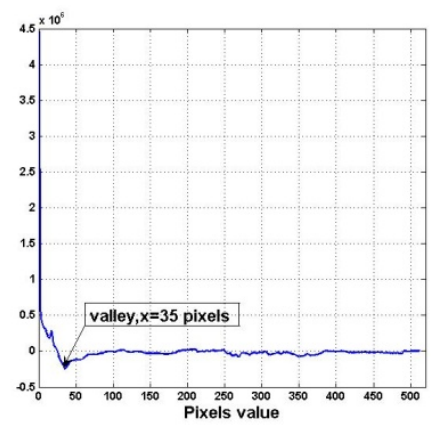

Fig. 7 Blur extent identification

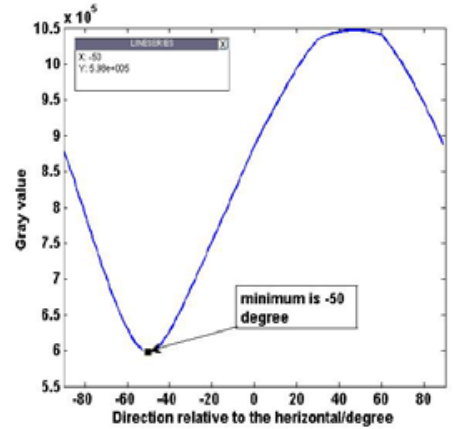

Fig. 8 Blur direction identification
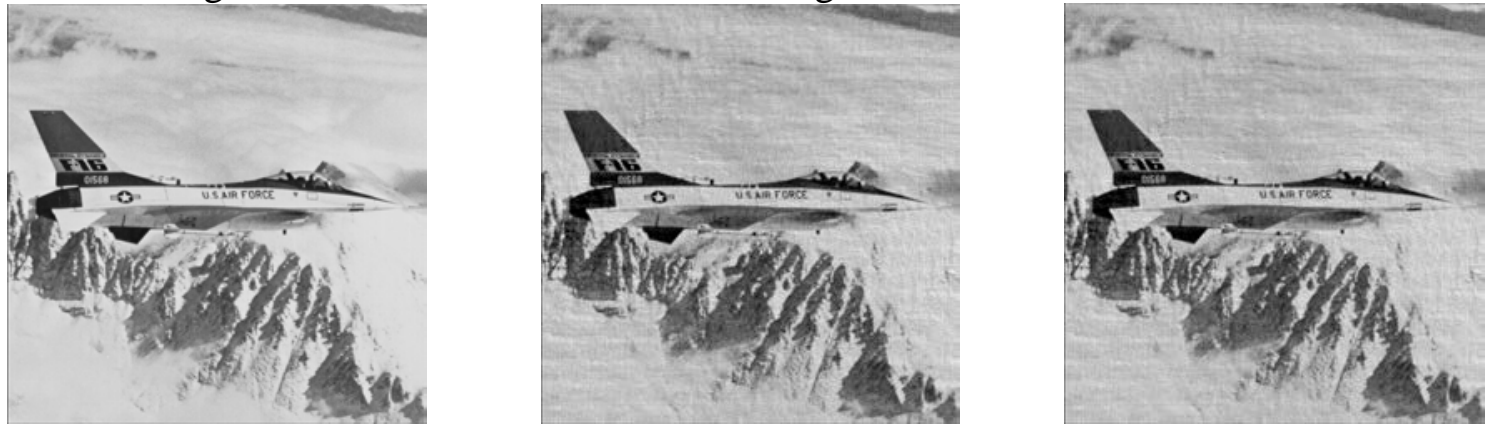

Fig. 9 Result of CLS algorithm Fig. 10 Result of BC algorithm Fig. 11 Result of LR algorithm

Table 2 Estimation of restored images

\begin{tabular}{|c|c|c|c|c|}
\hline $\begin{array}{c}\text { Estimation } \\
\text { methods }\end{array}$ & $\begin{array}{c}\text { Original } \\
\text { image }\end{array}$ & $\begin{array}{c}\text { Result of CLS } \\
\text { algorithm }\end{array}$ & $\begin{array}{c}\text { Result with 150 } \\
\text { iterations of BC } \\
\text { algorithm }\end{array}$ & $\begin{array}{c}\text { Result with 150 } \\
\text { iterations of LR } \\
\text { algorithm }\end{array}$ \\
\hline $\begin{array}{c}\text { Space } \\
\text { frequency }\end{array}$ & 17.2845 & 17.3021 & 24.7582 & 24.7237 \\
\hline Entropy & 0.0002 & 0.0087 & 0.0112 & 0.0103 \\
\hline $\begin{array}{c}\text { Standard } \\
\text { deviation }\end{array}$ & 46.4078 & 46.3272 & 46.9376 & 46.8322 \\
\hline Mean gradient & 5.4795 & 7.0079 & 8.9463 & 8.9069 \\
\hline
\end{tabular}

The original clear image adopted by the experiment is as shown in the Fig. 5, Fig. 6 represents its non-noise motion blur image, the real motion blur direction and extent are $-50^{\circ}$ and 35 pixels respectively. The identification results of the PSF's parameters tested by the proposed method are $-50^{\circ}$ and 35 pixels respectively, which are shown in the Fig. 7 and Fig. 8 . Based on the estimated PSF parameters, the paper adopts CLS, BC and LR algorithms, which are regularly used in blind image restoration field, to restore the blurred image(the iterations of BC and LR algorithm are both taken 150), the restored results are as shown in the Fig. 9-Fig. 11.From the analysis of test, we can discover that all of the restoration algorithms can get satisfied results and reach a conclusion that the proposed PSF estimated method can be generally applied to the restoration algorithms and the results possess higher accuracy.

\section{Conclusion}

Aiming at the restoration of blurred image, this paper proposes an improved estimation method of blurred image's PSF, the method comprehensively applies the direction differential operator and differential autocorrelation function, it can precisely estimate the vital parameters of the uniform linear motion model's PSF: blur direction and blur extent. On the base of estimated PSF, aiming at testifying the applicability and accuracy of this method, the paper adopts CLS, BC and LR algorithms to restore the non-noise blurred image and analyze the results. The experiments demonstrate that the 
kind of blind restoration algorithms based on the proposed estimated PSF can effectively restore the blurred images.

\section{References}

[1] ZHU Yi, QIN Shi-yin, Analysis of motion blur characteristics and its parameter estimation for space-based image [J]. Microcomputer Information, 24(10-3), 2008, 290-292.

[2] W H Richardson, Bayesian-based iterative method of image restoration [J]. JOSA, 62, 1972, 55-59.

[3] L B Lucy, An iterative technique for the rectification of observed distributions [J]. The Astronomical Journal, 79(6), 1974, 745-754.

[4] R C Gonzalez, R E Woods, Digital image processing (Third Edition) [M]. Publishing Home of Electronics Industry.

[5] CHEN Qianrong, LU Qisheng, CHENG Lizhi, Identification of the motion blurred direction of motion blurred images [J]. Journal of National University of Defense Technology, 26(1), 2004, 41-45.

[6] Y Yitzhaky, N S Kopeika, Identification of blur parameters from motion blurred images [J]. CVGIP: Graphical Models and Image Processing, 59(5), 1997, 310-320.

[7] Y Yitzhaky, R Milberg, S Yohaev, et al, Comparison of direct blind deconvolution methods for motion-blurred images [J]. Applied Optics, 38(20), 1999, 4325-4332. 